\title{
Standard radiographic assessments of distal radius fractures miss involvement of the distal radioulnar joint: a diagnostic study
}

\author{
Laura A. Hruby ${ }^{1} \cdot$ Thomas Haider $^{1,2} \cdot$ Roberta Laggner $^{1} \cdot$ Claudia Gahleitner $^{1} \cdot$ Jochen Erhart $^{2} \cdot$ Walter Stoik $^{1}$. \\ Stefan Hajdu ${ }^{1} \cdot$ Gerhild Thalhammer ${ }^{1}$ (1)
}

Received: 13 October 2020 / Accepted: 20 January 2021 / Published online: 8 February 2021

(c) The Author(s) 2021

\begin{abstract}
Introduction Distal radius fractures account for one-fifth of all fractures in the emergency department. Their classification based on standard radiographs is common practice although low inter-observer reliabilities and superiority of computer tomography (CT) scanning in evaluation of joint congruency have been reported.

Materials and methods We retrospectively analyzed 96 displaced distal radius fractures scheduled for open reduction and internal fixation using standard radiographic assessment. The radiographs were classified with the Arbeitsgemeinschaft für Osteosynthesefragen/Orthopaedic Trauma Association (AO/OTA), Fernandez and Frykman classifications by three observers and inter-rater reliabilities were calculated. Additional CT scanning was performed in all cases and the following parameters were assessed: radiocarpal joint involvement, fracture extent into the radial sigmoid notch, i.e. the distal radio-ulnar joint, comminution of the metaphysis, and concomitant ulnar styloid fracture. The CT scans were used as a reference standard to determine sensitivity and accuracy of standard radiographic assessment in evaluation of distal radius fractures.

Results The inter-rater agreement for the AO classification was 35.4\%, 68.8\% for the Fernandez and 38.5\% for the Frykman classification. Fracture extension into the radiocarpal joint was present in 81 cases (84.4\%). Sigmoid notch involvement was found in 81 fractures (84.4\%). Involvement of both joints was present in 72 cases (75\%). The sensitivity of standard radiographs regarding radiocarpal joint involvement was $93.8 \%$. Considering involvement of the distal radio-ulnar joint the false-negative rate using standard radiographs was $61.7 \%$ and the test's accuracy for sigmoid notch involvement was $45.8 \%$. Conclusion This study demonstrates that involvement of the sigmoid notch is frequently missed in standard radiographs. The presented data support the frequent use of CT imaging to allow the holistic illustration of a fracture's complexion and to ensure optimal pre-operative planning.
\end{abstract}

Keywords Distal radius fracture $\cdot$ Fracture classification system $\cdot$ Interobserver reliability $\cdot$ Distal radioulnar joint

\section{Background}

Distal radius fractures (DRFs) are among the most frequent injuries of the upper extremity and account for one-fifth of all fractures seen in the emergency department $[1,2]$. The acute management of DRFs depends on various clinical and radiological parameters [3]. For the initial assessment,

Gerhild Thalhammer

gerhild.thalhammer@meduniwien.ac.at

1 Department of Orthopedics and Trauma Surgery, Medical University of Vienna, Spitalgasse 23, 1090 Vienna, Austria

2 Department of Orthopedics and Traumatology, Hospital of the St. John of God Brothers Eisenstadt, Johannes von Gott-Platz 1, 7000 Eisenstadt, Austria two-plane radiographs remain the gold standard. However, precise evaluation in regards to intra-articular gaps and step-offs is limited with conventional radiography and computed tomography (CT) scans are known to allow for a better assessment of fracture complexion and extensions into articular surfaces [4].

Traumatic lesions of the distal radio-ulnar joint (DRUJ) occur in conjunction with fractures of the distal radius in a considerable number of cases and underappreciated injuries are a common cause of ulnar-sided wrist pain and limited range of motion [5-7]. Amongst the most common classification systems of distal radius fractures, however, only the Frykman classification [8] takes into account the involvement of the distal radio-ulnar joint. Few studies with limited patient numbers have compared distal radius fracture 
extensions using conventional radiographs and computer tomography (CT) [9-11], all of which found an underestimation of distal radio-ulnar joint involvement based on plain radiographs.

The purpose of this study was to compare fracture types and complexion of DRFs in standard radiographs and computed tomography (CT) scans. It was hypothesized that standard radiographic examinations underestimate involvement of both the radiocarpal and distal radioulnar joints.

\section{Methods}

Between February 2017 and January 2020, radiographic and $\mathrm{CT}$ examinations in patients with distal radius fractures were obtained in 96 consecutive cases. All included patients were scheduled for surgical treatment, i.e. open reduction and internal fixation (ORIF) with volar plate osteosynthesis. The standardized radiographic assessment of the wrist consisted of posteroanterior and lateral projections. The CT scans (Siemens Somatom Edge plus, Siemens Healthineers, Germany) were performed in prone position with the arm stretched forward over the head with forearm and wrist in neutral position. Evaluation of CT scans included sagittal, coronal and axial reconstructions in the bone window. Standard radiographic projections were obtained at initial consultation after injury as well as after closed reduction with the forearm immobilized in a cast. The timing of the CT scan was not standardized and ranged from immediately post reduction up to fifteen days after injury with the forearm immobilized in a cast.

Three observers independently evaluated anonymized radiographs and classified the fractures according to the AO/OTA [12], Fernandez [13] and Frykman [8] classification systems. The three observers were an orthopedic trauma surgery resident at the beginning of training, one at the end of training, as well as an orthopedic trauma surgery attending with specialization in hand surgery with over 15 years of clinical experience. No consensus was negotiated throughout the assessment of conventional radiographs. During evaluation of the digital images, schematic drawings of the respective classification systems were available on site for each observer.

Since additional CT scans were shown not to increase reliability of the mentioned classification systems $[9,10]$, the CT scans were evaluated by all 3 investigators holding consensus meetings. Each fracture was evaluated blinded in a randomized order. Axial, coronal, and sagittal reconstructions through both articular surfaces were evaluated in all cases. The following parameters were discussed and assessed: (1) radiocarpal joint involvement, (2) fracture extent into the radial sigmoid notch, i.e. the distal radioulnar joint, (3) comminution of the metaphysis, and (4) concomitant ulnar styloid fracture. To facilitate comparability of the results in terms of absolute and relative frequencies, the CT scans were also classified using the three mentioned classification systems. Since it was hypothesized that the experienced hand-specialized trauma surgeon would most accurately identify the true fracture morphology on plain radiographs, the $\mathrm{CT}$ images were compared to this observer's results.

To elucidate differences in correct fracture assessment related to clinical experience, results between observer 1, i.e. the orthopedic trauma surgery resident at the end of training, and observer 2, i.e. the orthopedic trauma surgery attending with specialization in hand surgery with over 15 years of clinical experience, were directly compared.

\section{Statistics}

Data were analysed using the statistical program $\mathrm{R}$ (version 3.6.1). Absolute and relative frequencies of all fractures are given. To describe inter-rater reliability of the respective fracture classification systems agreements are presented as percentage values. Sensitivity, false-negative rate and accuracy of radiographic assessments were calculated using CT scans as the true reference.

\section{Results}

\section{Study participants}

Ninety-six patients with DRF scheduled for ORIF with a mean age of $55 \pm 11$ years (range, 21-75) were included. Seventy-seven patients $(80 \%)$ were female.

\section{Fracture types comparing standard radiographs and CT scans}

Table 1 gives absolute values and relative frequencies of the classified fractures comparing conventional radiographs and computer tomography scans. In 15 cases (15.6\%), extraarticular fractures were diagnosed with the CT, while the radiocarpal joint alone was affected in 9 patients $(9.4 \%)$. Fracture extensions into the sigmoid notch occurred in 81 cases $(84.4 \%)$, while this was combined with radiocarpal joint involvement in 72 cases (75\%). In total, the radiocarpal joint was affected in 81 cases $(84.4 \%)$.

To outline differences in assessing radiocarpal joint involvement using plain radiographs and CT scans for a total of 96 cases, fourfold tables for two observers are shown (observer 1, i.e. an orthopedic trauma surgeon resident at the end of training; observer 2, i.e. a hand-specialized surgeon; see Table 2). While observer 1 correctly identified involvement of the radiocarpal joint using standard radiographs in 
Table 1 Absolute frequencies of the fracture distributions comparing standard radiographs (R) and computer tomography scans $(\mathrm{CT})$

\begin{tabular}{lrr}
\hline & $\mathrm{R}$ & $\mathrm{CT}$ \\
\hline $\mathrm{AO}$ & & \\
$\mathrm{A} 1$ & 0 & 0 \\
$\mathrm{~A} 2$ & 2 & 0 \\
$\mathrm{~A} 3$ & 14 & 15 \\
$\mathrm{~B} 1$ & 0 & 0 \\
$\mathrm{~B} 2$ & 0 & 0 \\
B3 & 4 & 1 \\
C1 & 19 & 1 \\
C2 & 43 & 35 \\
C3 & 14 & 44 \\
Fernandez & & \\
I & 16 & 15 \\
II & 4 & 1 \\
III & 76 & 78 \\
IV & 0 & 1 \\
V & 0 & 1 \\
Frykman & & \\
I & 3 & 1 \\
II & 11 & 5 \\
III & 20 & 5 \\
IV & 29 & 4 \\
V & 0 & 2 \\
VI & 3 & 7 \\
VII & 10 & 19 \\
VIII & 20 & 53 \\
\hline & &
\end{tabular}

$86.4 \%$ of all CT-verified articular fractures, the radiographic assessment's sensitivity to recognize articular involvement increased to $93.8 \%$ for observer 2. Figure 1 shows a case, where radiocarpal joint involvement was missed in plain radiographs and apparent in the coronal and axial CT reconstructions.

The distribution of recognized fracture extensions into the DRUJ comparing radiographs and CT scans was analyzed using the Frykman classification, since this is the only classification evaluating its involvement. Creating fourfold tables, the results for the same two observers were compared (see Table 3). The sensitivity of the standard radiographic assessment to identify DRUJ involvement was $33.3 \%$ for observer 1 and $38.3 \%$ for observer 2. When CT scans were considered as the reference, the false-negative rate of standard radiographs for DRUJ involvement therefore was $66.6 \%$ and $61.7 \%$, respectively, and the test's accuracy was $39.6 \%$ and $45.8 \%$, respectively. Figure 2 shows a case, where extension of the fracture into the sigmoid notch was not seen on standard radiographs, however, was obvious when coronal and axial CT reconstructions were evaluated.

Regarding comminution of the metaphysis, underestimation of a multi-fragmentary fracture occurred in 23 of 96 cases $(24.0 \%)$ using standard radiographs. Osseous avulsion of the ulnar styloid process was underappreciated in 12 of 96 cases $(12.5 \%)$.

\section{Reliability of the AO/OTA, Fernandez and Frykman classifications using standard radiographs}

The agreement for all observers was $35.4 \%$ for the AO/OTA classification, $68.8 \%$ for the Fernandez classification, and $38.5 \%$ for the Frykman classification. For clustering fracture types only into $\mathrm{A}, \mathrm{B}$ and $\mathrm{C}$ types, absolute agreement increased to $69.8 \%$ for the AO/OTA classification. Regarding the Frykman classification, in $56.2 \%$ of the cases agreement among all three observers was obtained regarding distal radio-ulnar joint involvement.

\section{Discussion}

Conventional radiographs in two planes remain the gold standard in assessing distal radius fractures in the emergency department. Using only plain radiographs, however, it is hardly possible to recognize the details of a complex fracture as multiple bone fragments overlap in one plane

Table 2 To compare radiocarpal joint involvement using radiographs (R) and computer tomography scans (CT) the AO/OTA classification (A, $\mathrm{B}, \mathrm{C})$ was used

\begin{tabular}{llcr}
\hline Observer 1 & CT $(\mathrm{B}+\mathrm{C})$ & $\mathrm{CT}(\mathrm{A})$ & \\
\hline $\mathrm{R}(\mathrm{B}+\mathrm{C})$ & 70 & 5 & 75 \\
$\mathrm{R}(\mathrm{A})$ & 11 & 10 & 21 \\
& 81 & 15 & 96 \\
\hline Observer 2 & $\mathrm{CT}(\mathrm{B}+\mathrm{C})$ & $\mathrm{CT}(\mathrm{A})$ & 80 \\
\hline $\mathrm{R}(\mathrm{B}+\mathrm{C})$ & 76 & 4 & 16 \\
$\mathrm{R}(\mathrm{A})$ & 5 & 11 & 96 \\
& 81 & 15 & 96 \\
\hline
\end{tabular}

Fourfold tables of the results for observers 1 and 2 are shown. "(A)" include extra-articular fractures, while "(B+C)" comprise intra-articular fractures 
Fig. 1 Distal radius fracture in a 63-year-old female patient. Considering the posteroanterior (a) and lateral (b) projections of plain film radiography, this fracture was classified as an extraarticular A2 fracture according to the AO/OTA classification. In the coronal (c) and axial (d) CT reconstructions, however, radiocarpal joint involvement was clearly identified
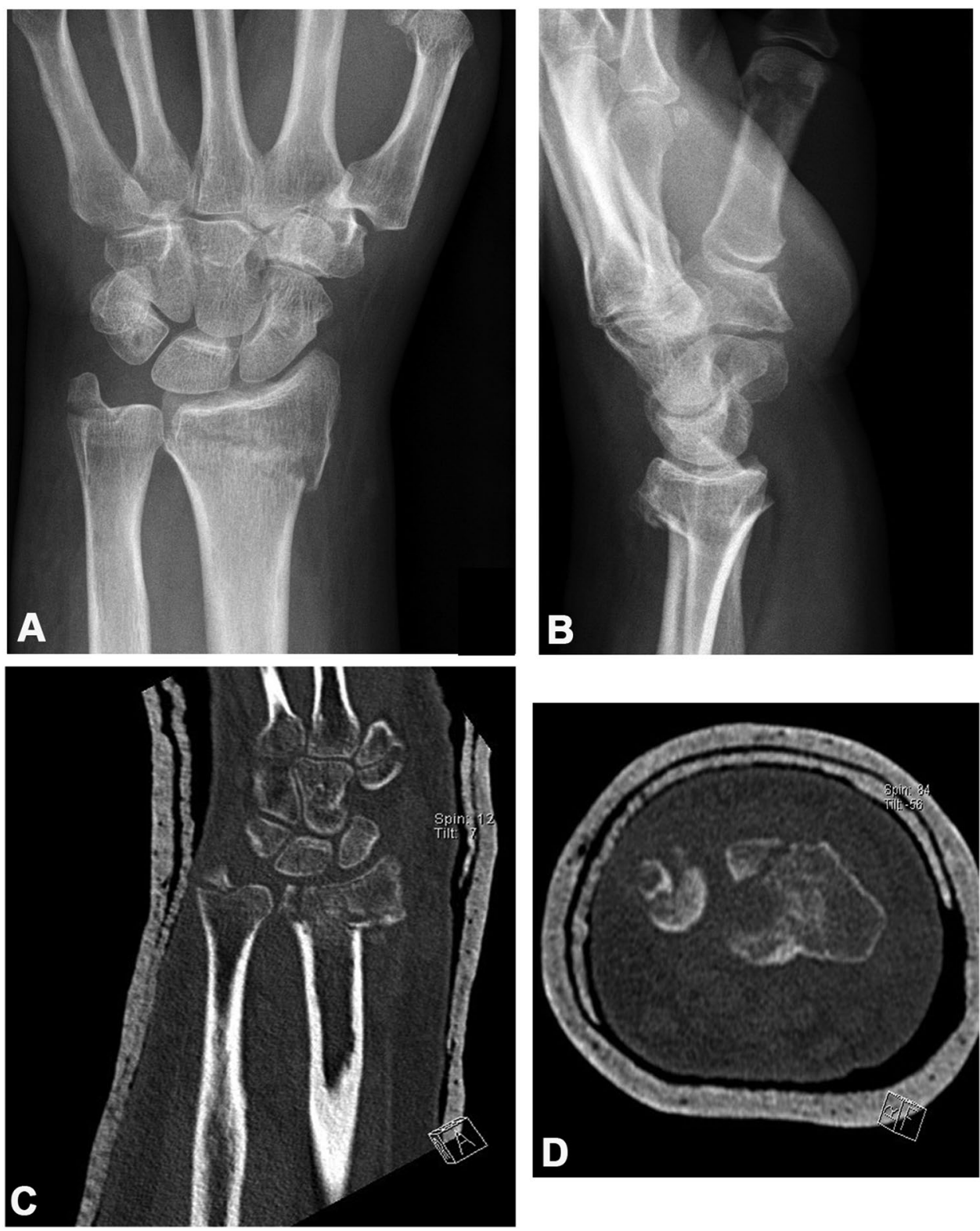

Table 3 To compare distal radio-ulnar joint (DRUJ) involvement using radiographs (R) and computer tomography scans (CT), the Frykman classification (I-VIII) was used

\begin{tabular}{llll}
\hline Observer 1 & CT (V-VIII) & CT (I-IV) & \\
\hline R (V-VIII) & 27 & 4 & 31 \\
R (I-IV) & 54 & 11 & 65 \\
& 81 & 15 & 96 \\
\hline Observer 2 & CT (V-VIII) & CT (I-IV) & 33 \\
\hline R (V-VIII) & 31 & 2 & 63 \\
R (I-IV) & 50 & 13 & 96 \\
\hline
\end{tabular}

Fourfold tables of the results for observers 1 and 2 are shown. "(I-IV)" comprise fractures not involving the DRUJ, whil (V-VIII) include fractures with DRUJ involvement 
Fig. 2 Distal radius fracture in a 61-year-old female patient. Using standard posteroanterior (a) and lateral (b) radiographs, this fracture was classified as a type IV fracture according to the Frykman classification. The coronal (c) and axial (d) CT reconstructions, however, into the sigmoid notch clearly show fracture extension
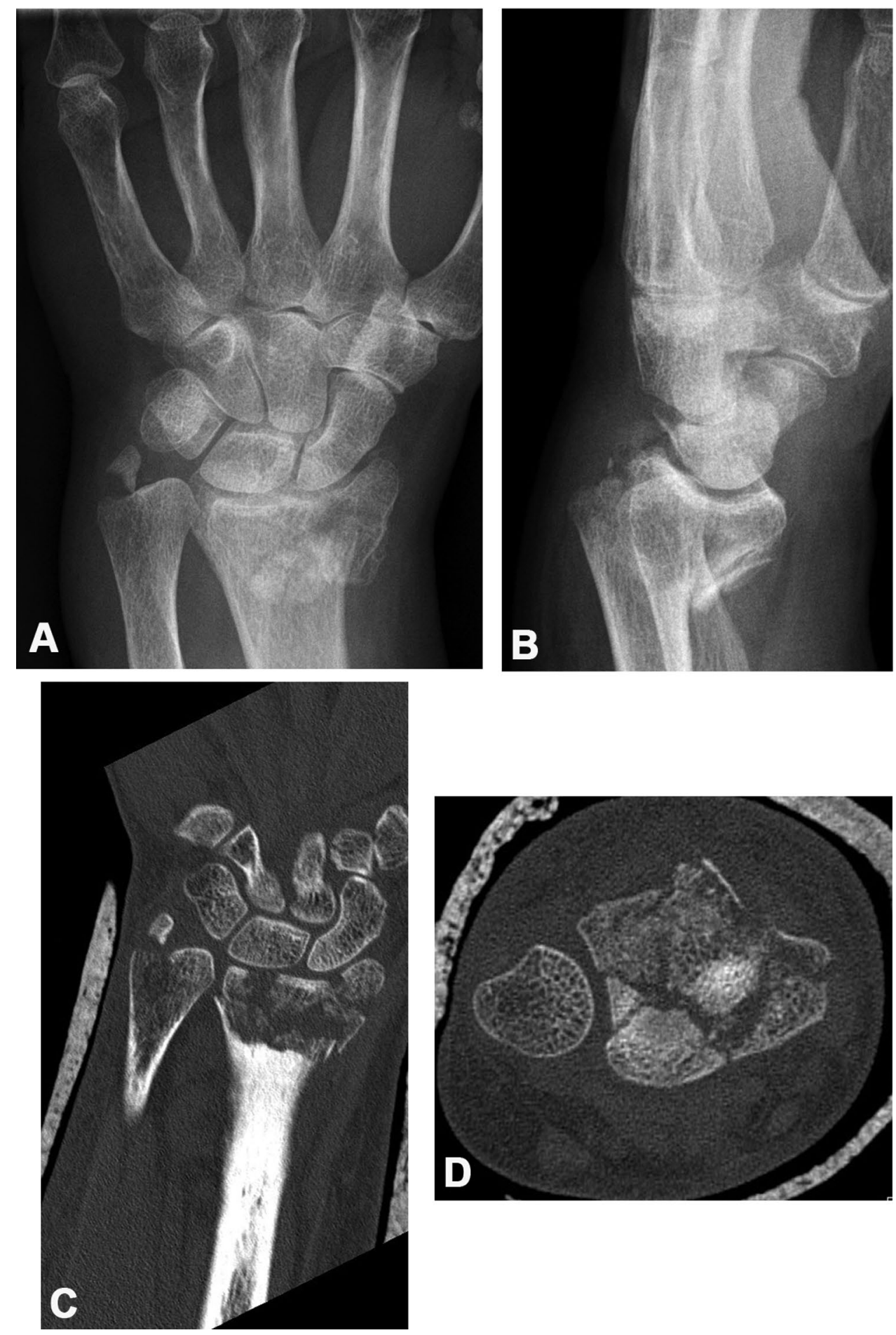

and the precise evaluation of gaps and step-offs in joint surfaces is inaccurate [4]. Also, DRFs in the acute setting are often associated with imperfect projection planes due to pain and concomitant loss of active and passive wrist motion. The superiority of CT scanning in evaluation of distal radius fractures has been shown by a number of authors [4, 14-16]. Still, however, its application in clinical decision-making, i.e. conservative versus operative treatment, as well as in pre-operative planning is not universally accepted [17]. The prerequisite of a useful classification system lies in the organization of clinical information in a manner that is consistent from one observation to the next [18]. However, the commonly applied systems, i.e. the AO/OTA, Frykman and Fernandez 
classifications, fail to fulfill these criteria. Poor inter- and intra-observer reliabilities have been shown for all mentioned classifications in various studies $[10,11,17,19$, 20]. The presented results of percent agreement amongst the three observers evaluating plain radiographs perfectly align with previously published results [9, 17-21]. As mentioned above, image quality of conventional radiographs greatly varies due to inaccurate projections and could possibly explain the low reliability of the common fracture classifications. Interestingly, several authors have shown that the inter-observer reliability of the various fracture classifications is not improved using additional computer tomography scanning [10]. It has to be noted that the original AO classification was originally designed for study purposes and neither of the mentioned classification systems were initially designed to evaluate fracture types with CT imaging. When axial, coronal and sagittal planes are incorporated in the fracture type evaluation a more accurate or rather "true" representation of the respective fracture may be recognized. Kleinlugtenbelt et al. [10] therefore concluded that the additional value of CT scanning over standard radiographic assessment is limited with regard to fracture classification reliability, but has significant implications for accurate evaluation of the fracture types. Flikkilä and colleagues [9], who also assessed the use of CT in regards to fracture classification using the AO/OTA system, came to a similar conclusion: $\mathrm{CT}$ is of minor value in improving the inter-observer reliability but offers much higher accuracy in evaluating joint involvement. In this study, CT images were analyzed in light of four distinct questions, i.e. radiocarpal and distal radio-ulnar joint involvements, metaphyseal comminution, and ulnar styloid fracture. Regarding radiocarpal joint involvement, the radiographic assessment's sensitivity was $86.4 \%$ for an orthopaedic trauma surgeon at the end of training and $93.8 \%$ for a hand-specialized surgeon with over fifteen years of clinical experience. The sensitivity of conventional radiographic assessment regarding distal radio-ulnar joint involvement, however, dropped to $33.3 \%$ and $38.3 \%$, respectively, when CT scans were considered as the true reference. Correct identification of fracture extensions into the DRUJ therefore did only negligibly improve with clinical experience and were missed in 61.7 to $66.6 \%$ of the cases. Generally, sigmoid notch involvement was found in $84.3 \%$ of all scanned fractures, which is similar or slightly higher than numbers from previous reports $[4,10,16,22]$. Generally, involvement of the sigmoid notch was best identified in axial CT reconstructions, as has been noted by other authors [23]. Upon fracture union of the distal radius, underappreciated injuries to the DRUJ are a common cause of ulnar-sided wrist pain [6]. Fractures extending into the radial sigmoid notch may disrupt the DRUJ complex at various levels, thereby altering biomechanics and kinematics of wrist and forearm motion $[16,24]$. Since motion in the DRUJ combines translational and rotational components, unappreciated injuries may therefore result in restricted pro- and supination [6, 25].

With the use of computed tomography, the severity of the assessed fractures increased significantly (see Table 1), e.g. in plain X-rays only 14 fractures were classified as multi-fragmentary, intra-articular C3 fractures, whereas CT scans revealed radiocarpal articular surface destruction in 44 cases. This has implications on the decision process of treating this injuries with regard to preventing posttraumatic arthritis. Knirk and Jupiter described already in 1986 [26] that post-traumatic arthritis was seen in patients with an incongruity of the articular surface with a step-off of two millimeters or more. They concluded that achieving and maintaining congruity of the articular surface of the distal part of the radius is paramount for impeding post-traumatic arthritis. In another study about arthritispredicting factors in distal intraarticular radial fractures, Lutz et al. [27] showed that an increased articular cavity depth should be avoided to prevent degenerative arthritis.

Considering the underestimation of radiocarpal and distal radioulnar joint involvement seen on plain radiographs, the presented results also signify the generous indication for CT scanning in fractures, where conservative treatment is initially anticipated. More severe or rather complex fracture types are to be expected in light of the presented results. Some studies showed that in elderly patients these findings seem to have no influence on wrist function [28, 29], but the clinical relevance in regards to facilitating the decision process of conservative versus surgical treatment especially in younger patients remains to be investigated.

Standard radiographic evaluation demonstrated that fracture involvement of either the DRUJ or the radiocarpal joint rendered fracture extension into the respective other articular compartment very likely. This correlation might be helpful as a rule of thumb in evaluation of DRF in projection radiography.

In a diagnostic study by Bombaci and colleagues [30], the authors obtained standard radiographs and magnetic resonance imaging (MRI) scans in patients with intraarticular DRFs and were able to show that the triangular fibrocartilaginous complex (TFCC) was disrupted in $45 \%$ of all cases. With Frykman fracture types VI and VIII the likelihood of a TFCC lesion was significantly higher compared to other fracture subtypes [30]. In the presented analysis, the absolute frequency of Frykman type VI and VIII fractures increased from 23 to 60 cases with the additional information from the CT scan, rendering a TFCC lesion highly likely in a considerable subset of distal radius fracture patients scheduled for ORIF. Although CT scans do not allow evaluation of ligamentous pathologies in the wrist, the complexion of the fracture is more accurately 
depicted and allows conclusions about concomitant injuries as oppose to standard radiographs.

\section{Study limitations}

As oppose to previous studies [16], intra-articular step-offs and gaps were not quantified within joint surfaces in this study, since the timing of the performed CT scan was not standardized and ranged from immediately post-reduction to fifteen days after injury, with the forearm immobilized in a cast in each case. False "negative" displacement might have been assessed immediately after closed reduction, while after a few days, secondary displacement of truly unstable fractures might still have occurred and delineated incongruences of the respective joints. As of today, the implication of non-displaced intra-articular fractures on functional outcomes remains unclear [10]. Although increased rates of post-traumatic wrist osteoarthritis evaluated with standard radiography have been reported following intra-articular distal radius fractures, these radiological findings did not affect functional outcomes nor patient satisfaction [31, 32]. The clinical relevance of intra-articular fracture extensions into the radiocarpal and distal radio-ulnar joint therefore remains to be investigated.

Only imaging data of patients with distal radius fractures who received surgical treatment were included in this study. Radiographic and computer tomography images of patients who were treated conservatively in a cast were not evaluated in regards to classification mismatch. Since DRFs treated conservatively usually represent milder forms of fracture types and rarely involve intra-articular, multi-fragmentary, or comminuted fractures, the applied classification systems might have shown higher agreements between radiographs and computer tomography scans in these cases.

\section{Conclusion}

Standard radiographic assessments do not allow sufficient evaluation of the articular congruity of the radiocarpal and distal radioulnar joints. To ensure the holistic illustration of a fracture's complexion, a CT scan is indispensable especially in intra-articular fractures as the severity is underestimated with plain X-rays.

\footnotetext{
Author contributions All authors contributed to the study conception. The study design was established by TH, JE, and GT. Material preparation, data collection and analysis were performed by LAH, TH, WS, RL, and GT. CG helped with the study design and performed all statistical analyses. The first draft of the manuscript was written by LAH. All authors commented on previous versions of the manuscript reviewing its content. All authors read and approved the final manuscript.
}

Funding Open Access funding provided by Medical University of Vienna.

\section{Compliance with ethical standards}

Conflict of interest The authors declare they have no financial interests.

Ethical approval The conducted study followed the principles of the Declaration of Helsinki and was approved by the local institutional review board (protocol number 2339/2016).

Informed consent All patients gave written informed consent to participate.

Open Access This article is licensed under a Creative Commons Attribution 4.0 International License, which permits use, sharing, adaptation, distribution and reproduction in any medium or format, as long as you give appropriate credit to the original author(s) and the source, provide a link to the Creative Commons licence, and indicate if changes were made. The images or other third party material in this article are included in the article's Creative Commons licence, unless indicated otherwise in a credit line to the material. If material is not included in the article's Creative Commons licence and your intended use is not permitted by statutory regulation or exceeds the permitted use, you will need to obtain permission directly from the copyright holder. To view a copy of this licence, visit http://creativecommons.org/licenses/by/4.0/.

\section{References}

1. Court-Brown CM, Caesar B (2006) Epidemiology of adult fractures: a review. Injury 37(8):691-697. https://doi.org/10.1016/j. injury.2006.04.130

2. Baron JA, Karagas M, Barrett J, Kniffin W, Malenka D, Mayor M, Keller RB (1996) Basic epidemiology of fractures of the upper and lower limb among Americans over 65 years of age. Epidemiology 7(6):612-618. https://doi.org/10.1097/00001648-19961 1000-00008

3. Walenkamp MM, Vos LM, Strackee SD, Goslings JC, Schep NW (2015) The unstable distal radius fracture-how do we define it? A Systematic Review J Wrist Surg 4(4):307-316. https://doi. org/10.1055/s-0035-1556860

4. Tanabe K, Nakajima T, Sogo E, Denno K, Horiki M, Nakagawa $R$ (2011) Intra-articular fractures of the distal radius evaluated by computed tomography. J Hand Surg Am 36(11):1798-1803. https ://doi.org/10.1016/j.jhsa.2011.08.021

5. Lindau T, Aspenberg P (2002) The radioulnar joint in distal radial fractures. Acta Orthop Scand 73(5):579-588. https://doi. org/10.1080/000164702321022884

6. Mespreuve M, Vanhoenacker F, Verstraete K (2015) Imaging findings of the distal radio-ulnar joint in trauma. J Belg Soc Radiol 99(1):1-20. https://doi.org/10.5334/jbr-btr.846

7. Spies CK, Langer MF, Müller LP, Unglaub F (2020) Reconstruction of the deep fibers of the distal radioulnar ligaments facilitating a tendon graft-Adams' procedure. Oper Orthop Traumatol 32(3):262-270. https://doi.org/10.1007/s00064-019-00638-7

8. Frykman G (1967) Fracture of the distal radius including sequelae-shoulder-hand-finger syndrome, disturbance in the distal radio-ulnar joint and impairment of nerve function. A clinical and experimental study. Acta Orthop Scand: Suppl 108:103. https ://doi.org/10.3109/ort.1967.38.suppl-108.01 
9. Flikkila T, Nikkola-Sihto A, Kaarela O, Paakko E, Raatikainen T (1998) Poor interobserver reliability of AO classification of fractures of the distal radius. Additional computed tomography is of minor value. The J Bone Joint Surg British 80(4):670-672. https://doi.org/10.1302/0301-620x.80b4.8511

10. Kleinlugtenbelt YV, Groen SR, Ham SJ, Kloen P, Haverlag R, Simons MP, Scholtes VAB, Bhandari M, Goslings JC, Poolman RW (2017) Classification systems for distal radius fractures. Acta Orthop 88(6):681-687. https://doi.org/10.1080/17453 674.2017.1338066

11. Dahlen HC, Franck WM, Sabauri G, Amlang M, Zwipp H (2004) Incorrect classification of extra-articular distal radius fractures by conventional X-rays. Comparison between biplanar radiologic diagnostics and CT assessment of fracture morphology. Der Unfallchirurg 107(6):491-498. https://doi.org/10.1007/s0011 3-004-0747-5

12. Müller ME, Nazarian S, Koch P, Schatzker J (1990) The comprehensive classification of fractures of long bones, vol 1. SpringerVerlag, Berlin Heidelberg, Berlin

13. Fernandez DL (2001) Distal radius fracture: the rationale of a classification. Chir Main 20(6):411-425. https://doi.org/10.1016/ s1297-3203(01)00067-1

14. Pruitt DL, Gilula LA, Manske PR, Vannier MW (1994) Computed tomography scanning with image reconstruction in evaluation of distal radius fractures. J Hand Surg Am 19(5):720-727. https:// doi.org/10.1016/0363-5023(94)90174-0

15. Johnston GH, Friedman L, Kriegler JC (1992) Computerized tomographic evaluation of acute distal radial fractures. J Hand Surg Am 17(4):738-744. https://doi.org/10.1016/03635023(92)90326-k

16. Rozental TD, Bozentka DJ, Katz MA, Steinberg DR, Beredjiklian PK (2001) Evaluation of the sigmoid notch with computed tomography following intra-articular distal radius fracture. J Hand Surg Am 26(2):244-251. https://doi.org/10.1053/jhsu.2001.22930

17. Kural C, Sungur I, Kaya I, Ugras A, Erturk A, Cetinus E (2010) Evaluation of the reliability of classification systems used for distal radius fractures. Orthopedics 33(11):801. https://doi. org/10.3928/01477447-20100924-14

18. Andersen DJ, Blair WF, Steyers CM Jr, Adams BD, el-Khouri GY, Brandser EA (1996) Classification of distal radius fractures: an analysis of interobserver reliability and intraobserver reproducibility. J Hand Surg Am 21(4):574-582. https://doi.org/10.1016/ s0363-5023(96)80006-2

19. Yinjie Y, Gen W, Hongbo W, Chongqing X, Fan Z, Yanqi F, Xuequn W, Wen M (2020) A retrospective evaluation of reliability and reproducibility of arbeitsgemeinschaftfur osteosynthesefragen classification and Fernandez classification for distal radius fracture. Medicine (Baltimore) 99(2):e18508. https://doi.org/10.1097/ MD.0000000000018508

20. Waever D, Madsen ML, Rolfing JHD, Borris LC, Henriksen M, Nagel LL, Thorninger R (2018) Distal radius fractures are difficult to classify. Injury 49(Suppl 1):S29-S32. https://doi.org/10.1016/ S0020-1383(18)30299-7

21. Arealis G, Galanopoulos I, Nikolaou VS, Lacon A, Ashwood N, Kitsis C (2014) Does the CT improve inter- and intra-observer agreement for the AO, Fernandez and Universal classification systems for distal radius fractures? Injury 45(10):1579-1584. https:// doi.org/10.1016/j.injury.2014.06.017
22. Gologan R, Ginter VM, Ising N, Kilian AK, Obertacke U, Schreiner U (2014) Carpal lesions associated with dislocated fractures of the distal radius. A systematic screening of 104 fractures using preoperative CT and MRI. Unfallchirurg 117(1):48-53. https://doi.org/10.1007/s00113-012-2264-2

23. Hintringer W, Rosenauer R, Pezzei C, Quadlbauer S, Jurkowitsch J, Keuchel T, Hausner T, Leixnering M, Krimmer H (2020) Biomechanical considerations on a CT-based treatment-oriented classification in radius fractures. Arch Orthop Trauma Surg 140(5):595-609. https://doi.org/10.1007/s00402-020-03405-7

24. Yan B, Chen Y, Yin W (2019) Influence of distal radius fractures involving the intermediate column on forearm rotation. J Orthop Surg Res 14(1):111. https://doi.org/10.1186/s13018-019-1155-4

25. Ishikawa J, Iwasaki N, Minami A (2005) Influence of distal radioulnar joint subluxation on restricted forearm rotation after distal radius fracture. J Hand Surg Am 30(6):1178-1184. https://doi. org/10.1016/j.jhsa.2005.07.008

26. Knirk JL, Jupiter JB (1986) Intra-articular fractures of the distal end of the radius in young adults. J Bone Joint Surg Am 68(5):647-659

27. Lutz M, Arora R, Krappinger D, Wambacher M, Rieger M, Pechlaner S (2011) Arthritis predicting factors in distal intraarticular radius fractures. Arch Orthop Trauma Surg 131(8):11211126. https://doi.org/10.1007/s00402-010-1211-3

28. He B, Tian X, Ji G, Han A (2020) Comparison of outcomes between nonsurgical and surgical treatment of distal radius fracture: a systematic review update and meta-analysis. Arch Orthop Trauma Surg 140(8):1143-1153. https://doi.org/10.1007/s0040 2-020-03487-3

29. Arora R, Lutz M, Deml C, Krappinger D, Haug L, Gabl M (2011) A prospective randomized trial comparing nonoperative treatment with volar locking plate fixation for displaced and unstable distal radial fractures in patients sixty-five years of age and older. $\mathbf{J}$ Bone Joint Surg Am 93(23):2146-2153. https://doi.org/10.2106/ JBJS.J.01597

30. Bombaci H, Polat A, Deniz G, Akinci O (2008) The value of plain X-rays in predicting TFCC injury after distal radial fractures. J Hand Surg Eur 33(3):322-326. https://doi.org/10.1177/17531 93408090106

31. Young BT, Rayan GM (2000) Outcome following nonoperative treatment of displaced distal radius fractures in low-demand patients older than 60 years. J Hand Surg Am 25(1):19-28. https ://doi.org/10.1053/jhsu.2000.jhsu025a0019

32. Lameijer CM, Ten Duis HJ, Dusseldorp IV, Dijkstra PU, van der Sluis CK (2017) Prevalence of posttraumatic arthritis and the association with outcome measures following distal radius fractures in non-osteoporotic patients: a systematic review. Arch Orthop Trauma Surg 137(11):1499-1513. https://doi.org/10.1007/ s00402-017-2765-0

Publisher's Note Springer Nature remains neutral with regard to jurisdictional claims in published maps and institutional affiliations. 